\title{
Comprehensive Analysis of the Association Between Human Non-obstructive Azoospermia and Plasticisers via Single-Cell and Traditional RNA Sequencing Methods
}

\author{
Xu Zhang ${ }^{1,2} \cdot$ Xiaohan Ren $^{1,2} \cdot$ Tongtong Zhang ${ }^{1,2} \cdot$ Xiang Zhou $^{1,2} \cdot$ Xinglin Chen $^{1,2} \cdot$ Hongcheng Lu $^{1,2} \cdot$ Xuan Zhou $^{1,2} \cdot$ \\ Xiaolei Zhang ${ }^{1,2}$. Shangqian Wang ${ }^{1,2}$. Chao Qin ${ }^{1,2}$
}

Received: 3 June 2021 / Revised: 11 November 2021 / Accepted: 20 December 2021 / Published online: 30 January 2022

(c) The Author(s) 2021

\begin{abstract}
A father's lifetime experience is a major risk factor for a range of diseases in an individual. The influences of exposure can also be transmitted to offspring. Previous studies demonstrated that plasticisers can damage the male offspring reproductive system, but the link between mammalian research and human non-obstructive azoospermia remains underexplored. Here, we analysed reproduction-related genes from four publicly available single-cell RNA-Seq datasets and RNA-Seq datasets on GEO databases to investigate the correlation between human non-obstructive azoospermia and exposure to plasticisers during pregnancy. The $\mathrm{R}$ software was used in statistical analyses. A total of 9 co-upregulated genes and 1 co-downregulated gene were found. The Gene Ontology enrichment analyses were performed using the "clusterProfiler" package. Single-cell analyses were conducted to visualise the gene expression pattern in testis cell subgroups. Immunohistochemical images were used to evaluate the level of protein in testicular organs from The Human Protein Atlas. GSVA results provided further functional annotations. Three signature genes, i.e. COL1A1, CYP17A1 and KIF11, might serve as promising diagnostic biomarkers in non-obstructive azoospermia caused by plasticisers. Our results provided a potential new protocol to evaluate the feasibility of life or clinical intervention in patients with non-obstructive azoospermia. We believe that these observations will improve our understanding of the potential mechanisms of plasticiser contributions to human non-obstructive azoospermia and help identify potential targets for immunotherapy in the future.
\end{abstract}

Keywords Plasticizer $\cdot$ Non-obstructive azoospermia $\cdot$ Bioinformatics

Xu Zhang, Xiaohan Ren, Tongtong Zhang and Xiang Zhou have contributed equally to this work.

Xiaolei Zhang

zx120141577@163.com

Shangqian Wang

wsq5501@126.com

$\triangle$ Chao Qin

nmuqinchao@163.com

1 Department of Urology, The First Affiliated Hospital of Nanjing Medical University, Nanjing 210029, China

2 State Key Laboratory of Reproductive Medicine, Nanjing Medical University, 101 Longmian Avenue, Nanjing 211166, China

\section{Introduction}

Given the increasing level of industrialisation and urbanisation, plastics, pesticides, plasticisers and synthetic drugs are widely used (Kasonga 2021). Although these materials have improved the certain quality of human life, increasing chemicals are released into the environment and cause potential harm to animals and humans through the food chain. Endocrine-disrupting chemicals (EDCs), which are exogenous substances that cause adverse health effects in undamaged organisms or changes in the endocrine function of the offspring of organisms, include artificial compounds, chemical pollutants, natural oestrogens, plants and fungi (Golshan and Alavi 2019; Li et al. 2021). Many EDCs have stimulating hormone activity, which can affect steroid metabolism and change the oestrogen/androgen balance in the body (Stewart 2020). After the twentieth century, plastic products develop rapidly due to their wide use, low price and 
durable characteristics. Plasticisers are a kind of polymer material additive that can increase the flexibility of a polymer. Given their versatility, robustness and low production costs, plastics are used in a wide variety of applications. Plasticisers are mixed with polymers to increase the flexibility of plastics. However, plasticisers are not covalently bound to plastics and leach from products into the environment. Di-(2-ethylhexyl)-phthalate (DEHP) is a compound used as a plasticiser that can easily leach from the plastic into the environment. Mono-(2-ethylhexyl) phthalate (MEHP) is the metabolite of DEHP (Kuroda et al. 2020). Trioctyl trimellitate (TOTM), a new plasticiser, may be the preferred plasticiser instead of DEHP for medical polyvinyl chloride instruments due to its good plasticising property, migration resistance, high temperature resistance and low toxicity (Sheikh et al. 2016).

The male semen quality is closely related to male reproductive health and have been paid attention by people (Rahban and Nef 2020). In the past few decades, many studies reported a decline in human sperm quality. Wang et al. demonstrated that many semen parameters of Chinese young men show a decreasing trend within 15 years (Wang et al. 2019). Lidia Mínguez-Alarcón et al. found that sperm parameters, such as sperm concentration, total sperm count, motility and morphology, have significantly decreased amongst subfertile men from 2000 to 2017 (Minguez-Alarcon et al. 2018). The decline in sperm quality is affected by many factors, including tobacco and alcohol use, irregular daily routine and environmental toxicants. Recently, many studies focussed on the effects of plasticiser on male sperm quality due to the wide exposure to plasticiser through the food chain. The distribution of plasticiser or their metabolites can inevitably be detected in various organs of the human body (Sun et al. 2020). Animal studies confirmed that exposure to DEHP phthalate during pregnancy can lead to the deterioration of sperm parameters and imbalance of sex hormone secretion in male rat offspring (Lucas et al. 2012). One in 100 men is estimated to have azoospermia, which is the complete lack of sperm in the ejaculate. Currently, $20 \%$ of azoospermia cases remain idiopathic. The non-obstructive azoospermia (NOA) is mostly explained by congenital factors leading to spermatogenic failure, such as chromosome abnormalities and gene mutation ${ }^{5}$. However, knowledge on the link between NOA and exposure to plasticisers during pregnancy remains lacking. Knowledge on the causes of NOA is limited. High genetic heterogeneity due to the complexity of spermatogenesis and testicular function, lack of nonconsanguineous familial cases and confirmatory studies challenge this field. In clinics, patients with NOA often need to be treated with testicular and epididymal incision and sperm extraction under a microscope, but the success rate is relatively low. Therefore, increasing attention has been paid to the study of the pathogenesis of NOA and the further exploration of prevention and treatment measures. Further studies confirmed the associations of exposure to plasticisers during pregnancy with reproductive hormones and semen parameters (Dobrzynska and Tyrkiel 2019). The positive relationship between sperm concentration and the metabolite of DEHP is found (Barakat et al. 2019). Besides, with increased DEHP metabolites found in urine, the concentrations of serum FSH and LH increase, indicating impaired function of the testis and infertility (Hauser 2008). Iman Al-Saleh et al. confirmed that men who excrete high levels of MEHP relative to the other DEHP metabolites may be at risk of having low sperm concentration (Al-Saleh et al. 2019).

In the present study, two gene chips are downloaded from the NCBI-Gene Expression Omnibus (GEO) database (GSE41901 and GSE9210 datasets) to search for differentially expressed genes (DEGs) in the testes of offspring exposed to plasticiser during pregnancy and human testes with NOA. The possible mechanism of plasticiser on the male spermatogenic function is explored. The Gene Ontology (GO) functional annotation analysis is applied. Immunohistochemical (IHC) and single-cell analyses are used to compare the expression of these key genes in testicular cells. We hope to explore the link between NOA and exposure to plasticisers during pregnancy. Corresponding biomarkers are found to guide the diagnosis of male infertility caused by the plasticiser. The decline in human sperm level is an indisputable fact. Besides, male reproductive health should be protected.

\section{Materials and Methods}

\section{Data Set Download}

Two transcription profile datasets (i.e. GSE41901 and GSE9210) were obtained from GEO databases (http://www. ncbi.nlm.nih.gov/geo/), an open functional genome dataset. The platform for GSE41901 was GPL7294 (Agilent-014879 Whole Rat Genome Microarray $4 \times 44$ K G4131F). The microarray data of GSE9210 were based on the GPL887 platform (Agilent-012097 Human 1A Microarray (V2) G4110B). The GSE41901 dataset included 20 foetal testis samples and rats exposed to DEHP, MEHP and TOTM in utero at doses of $500 \mathrm{mg} / \mathrm{kg}$ administered through daily oral gavage to pregnant dams between gestational days 12 and 19 . The RNA of their foetal testis was extracted. The GSE9210 dataset was based on genomic gene expression in testes of 47 patients with NOA and 11 patients with obstructive azoospermia (OA). Patients diagnosed with azoospermia underwent two semen examinations, and two centrifugal semen samples confirm the absence of sperm in the ejaculate. All patients underwent testicular sperm extraction (TESE) for 
diagnosis. OA is defined as motile sperm collected from microsurgical epididymal sperm aspiration, or a large number of mature sperm are found form testicular sperm extraction. NOA is defined as having no sperm from microsurgical epididymal sperm aspiration and testicular sperm extraction. In addition, azoospermia patients with varicocele, ejaculatory dysfunction and endocrinopathy were excluded (Okada et al. 2008). In addition, in order to verify the specificity of the screened genes as diagnostic biomarkers, two gene chips (GSE7755, GSE108194) were downloaded from GEO databases, which is correlated with cryptorchidism and human oligospermia. The GSE7755 dataset included 34 normal testis samples and 20 testis samples with cryptorchidism. The GSE108194 dataset was based on genomic gene expression of 18 patients in peripheral blood with at least two semen routine examinations showing sperm density lower than 15 million/ml and genomic gene expression of 6 normal people in peripheral blood.

\section{Processing of Dataets and Screening of DEGs}

We first downloaded the original comment file and TXT matrix file of the platform and used the original comment file of the platform to change the probe ID in the matrix to gene symbol ID through the Perl script. We divided the data of GSE41901 into two groups, i.e. control and exposure groups, to investigate their expression. The GSE9210 dataset was also divided into two groups (i.e. patients with NOA and OA). The R software (version 4.0.2, https://www.r-project. org/) was used in data mining and statistical analyses, and the LIMMA package was used to mine the mRNA of abnormal expression levels. Through the LIMMA analysis, genes with absolute $\operatorname{logFC}>0.8$ and $P$ value $<0.05$ were considered to be DEGs.

\section{GO and Pathway Enrichment Analyses}

The "clusterProfiler" package was used in R to perform the GO functional annotation pathway enrichment analysis and explore the biological and molecular functions of DEGs. The biological process (BP), molecular function (MF) and cellular component (CC) in the GO analysis were performed. $P<0.05$ was selected as threshold in the analysis. The Venn diagram was used to identify co-downregulated and coupregulated genes in patients with NOA and rat offspring with plasticiser exposure.

\section{Assessment of DEGs at the Single-Cell Transcriptional Level}

Two adult testis tissues of GSE124263 from the GEO dataset were used to visualise the expression patterns of co-downregulated and co-upregulated genes in testis cell subgroups and examine the correlation between co-expression genes and the normal testis tissue at the single-cell level. The selected cell samples contained different cell populations, including Sertoli cell, spermatogonium, Leydig cell, endothelial cell, myoid cell, primary spermatocyte, macrophage, elongated spermatid, differentiating spermatogonia and sperm. The Seurat R package was used to perform the single-cell analysis. The gene features in each cluster were found by "scanpy". The cell-cell interactions were calculated using the "SingleCellSignalR" package in R.

\section{IHC Detection in Testicular Tissue Sections}

IHC images were used to evaluate the level of co-downregulated and co-upregulated genes and proteins in testicular organs from The Human Protein Atlas website (https://www. proteinatlas.org/) and validate the different expression levels of co-expression genes in testis cells.

\section{Gene Set Variation Analysis (GSVA) of COL1A1, CYP17A1 and KIF11}

The R software (version 4.0.3, https://www.r-project.org/) was used on specific cells to explore BPs associated with KIF11-, COL1A1- and CYP17A1-positive cells. The gene set was c5.go.v7.2.symbols.gmt (https://www.gsea-msigdb. org/gsea/index.jsp). GSVA was applied to evaluate the enrichment scores for each cell. The GO enrichment analysis with absolute $\log \mathrm{FC}>0.3$ and $P$ value $<0.05$ were considered to be differentially GO functional pathway.

\section{Results}

\section{Screening of DEGs}

We used the RNA-seq data from the GEO database to compare the differential expression of two transcription profiles. After data download and preprocessing by using the Perl script, we manually grouped the GSE9210 dataset to search for DEGs between DEHP-, MEHP- and TOTM-exposure and control groups. Compared with those in the control group, 244 upregulated and 513 downregulated genes were found in DEHP-exposure groups, 377 upregulated and 1230 downregulated genes were found in MEHP-exposure groups and 413 upregulated and 1476 downregulated genes were found in TOTM-exposure groups. Similarly, we then compared the DEGs in the testis of patients with NOA and OA (control group), and results showed 1217 downregulated and 483 upregulated genes in the testis of patients with NOA compared with those in the testis of patients with OA. The standard was $P<0.05$ and absolute $\log 2 \mathrm{FC}>0.8$ (Fig. 1). 
(a)

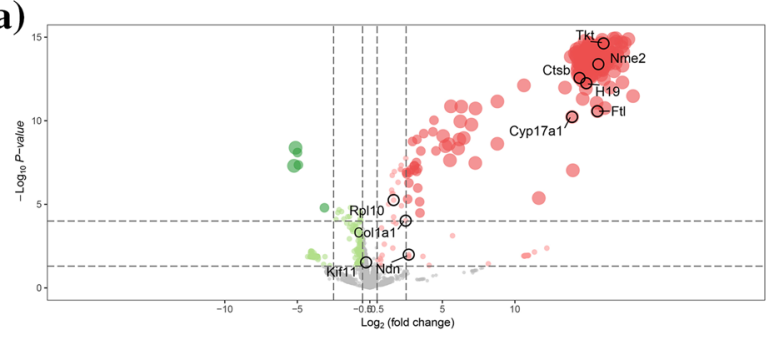

(c)

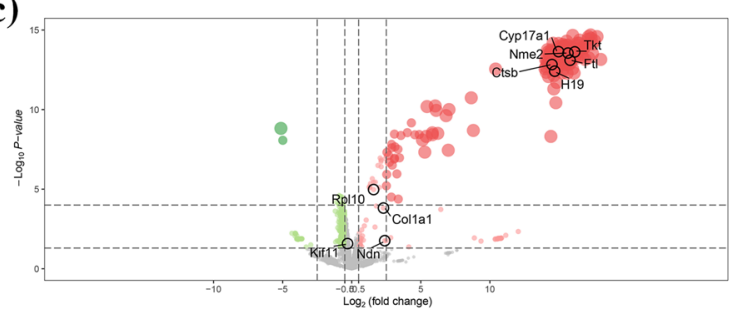

(b)

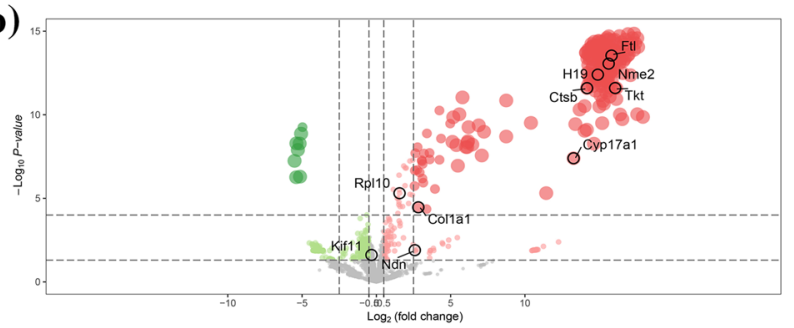

(d)

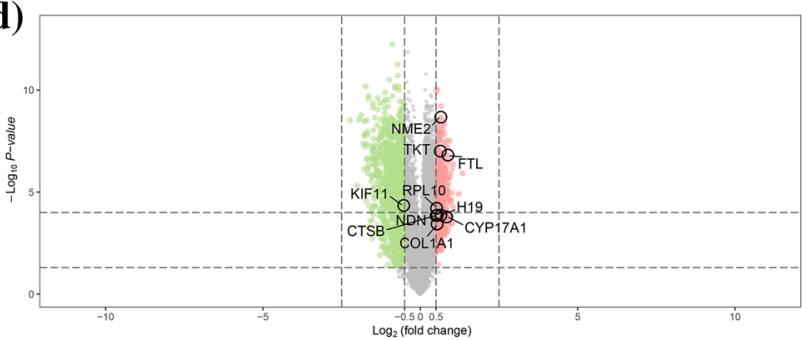

of 200 RNA-seq data was shown in the $-\log (P$ value) vs $\log$ (FoldChange) plot. The co-expression genes upregulated and downregulated groups were labelled with gene symbol ID

rough endoplasmic reticulum membrane. In MF (Fig. 4), DEGs were enriched in the structural constituent of ribosome and rRNA binding. The results of DEG functional analysis between OA and NOA showed that DEGs were enriched cellular processes involved in reproduction in multicellular organism, germ cell development, spermatid development and differentiation and meiotic cell cycle and fertilisation in BP. In CC, DEGs were enriched in acrosomal vesicle, motile cilium and collagen-containing extracellular matrix. In MF, DEGs were enriched in tubulin binding, nucleocytoplasmic carrier activity and microtubule motor activity (Fig. 5).

\section{Single-Cell Analysis of Co-upregulated and Co-downregulated Genes}

The tissues of testis contain several cell populations, such as Sertoli cell, spermatogonium, Leydig cell, endothelial cell, myoid cell, primary spermatocyte, macrophage, elongated spermatid, differentiating spermatogonia and sperm. To investigate the expression levels of co-upregulated and co-downregulated genes in human testicular tissues, we performed a single-cell analysis of the GSE124263 dataset. The UMAP method was used to perform nonlinear dimensional reduction. Cells were clustered in accordance with their cell type. The microsatellite status was also shown by the UMAP plot. For all co-upregulated genes, NME2, TKT, CTSB, FTL and RPL10 showed no significant difference in gene expression amongst different clusters. NBN and COL1A1 showed an enrichment in Sertoli, Leydig and myoid cells, whereas 

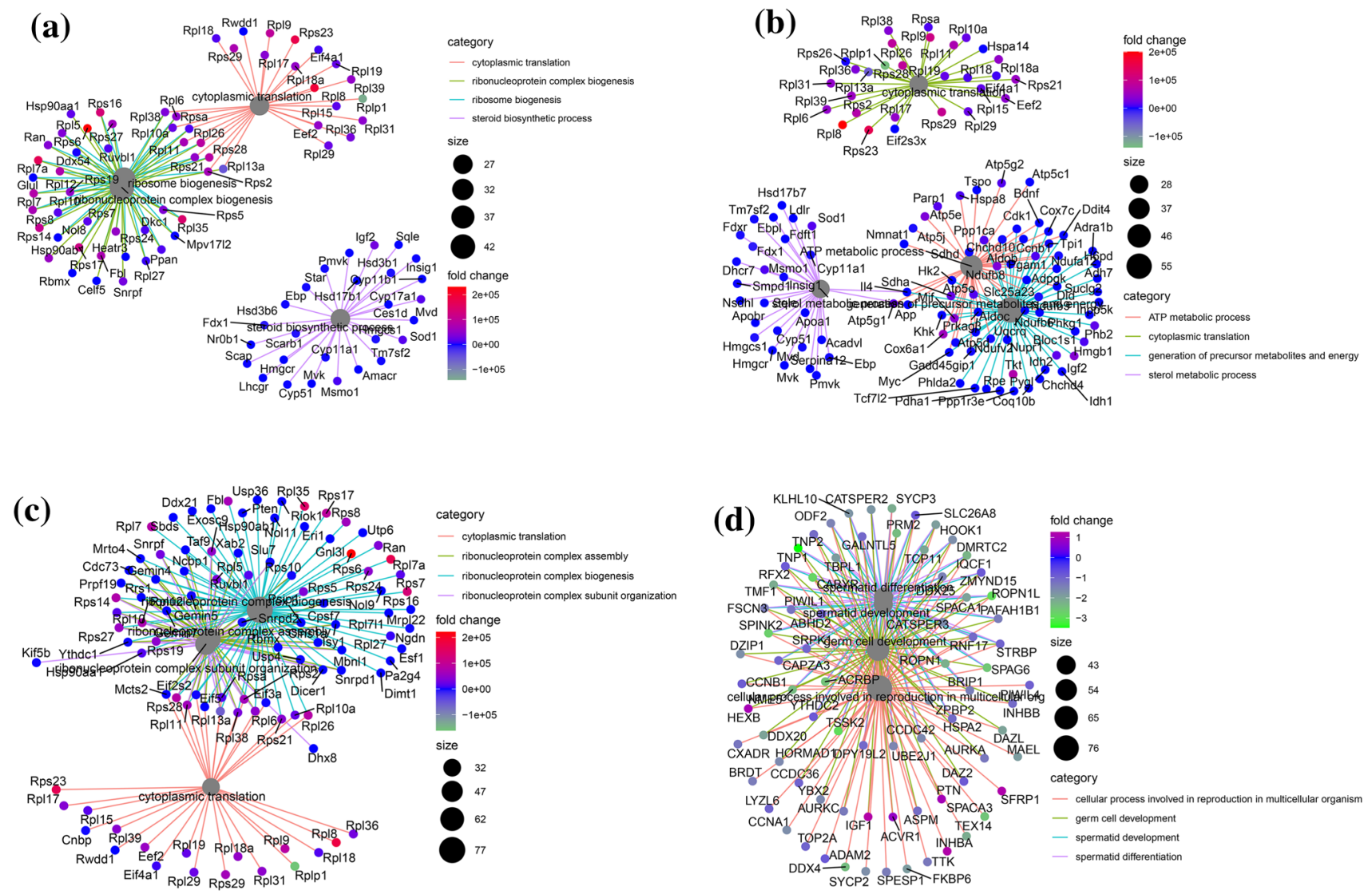

Fig. 2 Top 4 terms from the GO BP enrichment analysis. a DEHP-exposure group. b MEHP-exposure group. c TOTM-exposure group. d NOA group. The size of the dot represents counts, and the colour of the dot represents $P$ value

spermatogonium and primary spermatocyte showed no enrichment. Another co-upregulated gene, CYP17A1, was only enriched in Sertoli cell compared with another cluster. We also investigated the KIF11, a co-downregulated gene in the exposure group and patients with OA, and cell distribution patterns by the single-cell analysis. Interestingly, KIF11 was enriched in spermatogonium and primary spermatocyte (Figs. 6 and 7).

\section{IHC Detection of NBN, COL1A1, CYP17A1 and KIF11 in Testis Tissue Sections}

In HPA databases, we evaluated the mRNA and protein expression levels of NBN, COL1A1, CYP17A1 and KIF11 in the testis tissue. CYP17A1 was stably expressed in the testis tissue. Elevated staining for CYP17A1 was observed in the Leydig cell, whereas no staining was found in the seminiferous cell. Low staining for COL1A1 was observed in the Leydig cell, whereas moderate staining was observed in the seminiferous cell. IHC results revealed that NBN was highly expressed in the seminiferous cell and moderately expressed in the Leydig cell, and this finding was not consistent with the results of our single-cell analysis. KIF11, the only co-downregulated gene, was observed to be overexpressed in the seminiferous cell and had low expression in the Leydig cell (Fig. 8).

\section{Biological Relevance of COL1A1, CYP17A1 and KIF11}

We performed the GSVA of COL1A1, CYP17A1 and KIF11 to figure out the GO BP, CC and MF pathways in their overexpressed cells. GSVA showed enrichment of COL1A1, CYP17A1 and KIF11 in their overexpressed cells. In spermatogonia, the GO pathway analysis showed that cells with low expression of KIF11 were significantly enriched in cyclin binding, apoptotic process, toxic substance binding, biological adhesion, DNA replication and cell cycle compared with cells with high expression of KIF11. In the primary spermatocyte, the GO pathway analysis showed that cells with low expression of KIF11 were significantly enriched in cyclin binding, cell cycle, toxic substance binding, identical protein binding and nuclear outer membrane of the endoplasmic reticulum membrane network compared with cells with high expression of KIF11. The COL1A1 gene was upregulated in Sertoli and Leydig cells. In Sertoli cells, specific GO categories, such as exon-exon junction complex, 

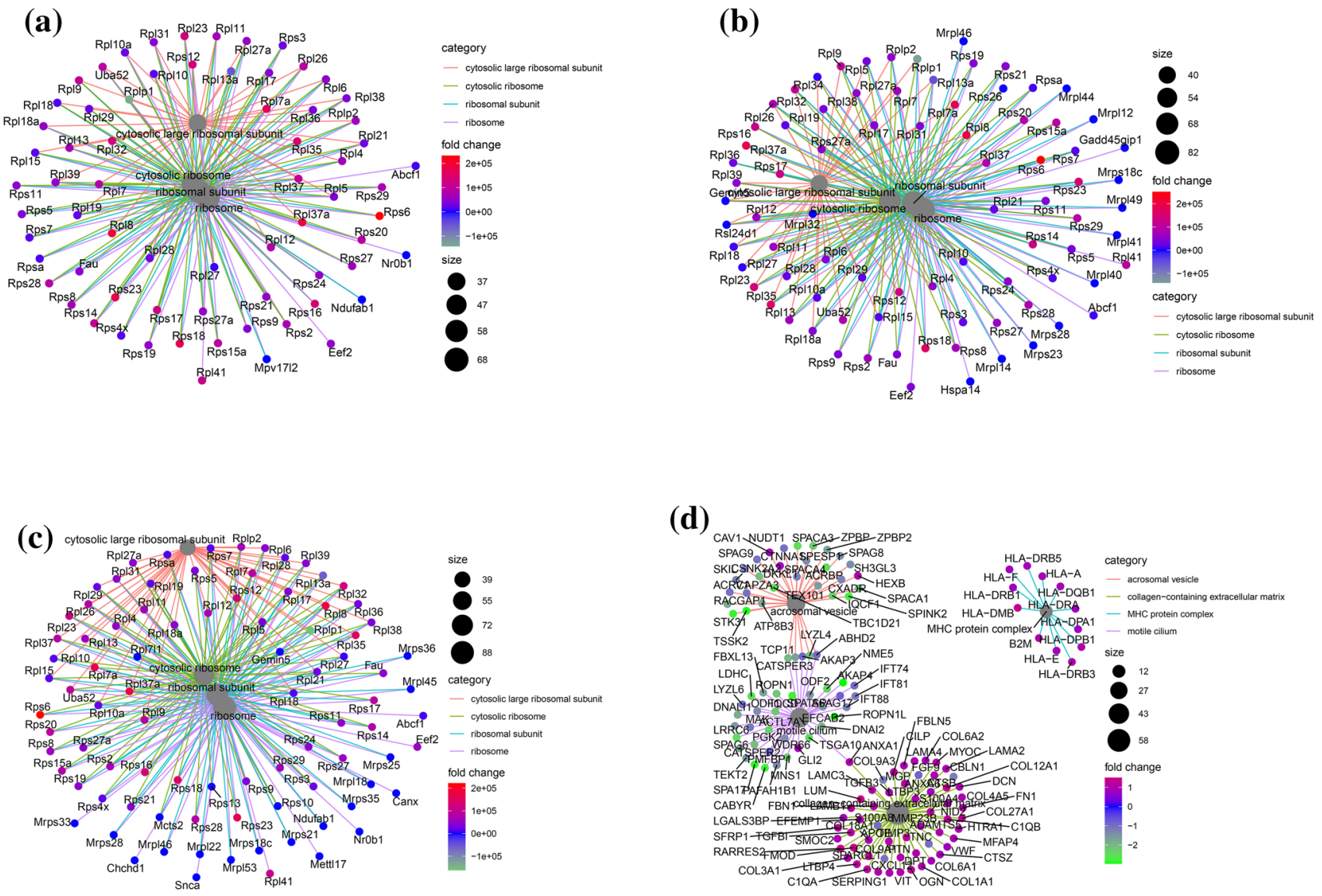

Fig. 3 Top 4 terms from the GO CC enrichment analysis. a DEHP-exposure group. b MEHP-exposure group. c TOTM-exposure group. d NOA group. The size of the dot represents counts, and the colour of the dot represents $P$ value

chaperone binding, RNA binding, cyclin binding and microtubule cytoskeleton, were significantly related to cells with high expression of COL1A1 than to cells with low expression of COL1A1. In Leydig cells, GO results showed that these cells with high expression of COL1A1 could participate in sequence-specific DNA binding, biological adhesion and protein dimerisation activity. Besides, in Sertoli cells, cells with high expression of CYP17A1 were enriched in kinase binding, misfolded protein binding and microtubule cytoskeleton compared with cells with low expression of CYP17A1 (Fig. 9).

\section{Exploration of the Role of COL1A1, CYP17A1 and KIF11 in Cryptorchidism and Human Oligospermia}

In order to verify the specificity of COL1A1, CYP17A1 and KIF11 as the diagnostic biomarkers for non-obstructive azoospermia caused by plasticisers, two transcription profile datasets (GSE7755, GSE108194) were obtained from GEO databases, which is correlated with cryptorchidism and human oligospermia. Then, the differential expressed analysis of GSE108194 dataset and GSE7755 dataset were performed with absolute $\log \mathrm{FC}>0.5$ and $P$ value $<0.05$. We obtained a total of 527 co-upregulated and 340 co-downregulated genes in the GSE108194 dataset. In the GSE7755 dataset, a total of 68 co-upregulated and 119 co-downregulated genes were observed. The Venn diagram demonstrated that COL1A1, CYP17A1 and KIF11 are not involved in the DEGs of GSE108194 dataset and GSE7755 dataset (Fig. 10). The results revealed that COL1A1, CYP17A1 and KIF11 are not correlated with other reproductive disorders, including cryptorchidism and human oligospermia.

\section{Discussion}

Infertility affects about 1 in 6 couples attempting pregnancy, and the man is responsible in approximately half of the cases. Given that the pathophysiology underlying azoospermia is not elucidated, most male infertility is diagnosed as idiopathic. With the recent azoospermia pandemic, male reproductive health, as one of the important reasons that cannot be ignored, has attracted increasing attention. At present, 
(a)

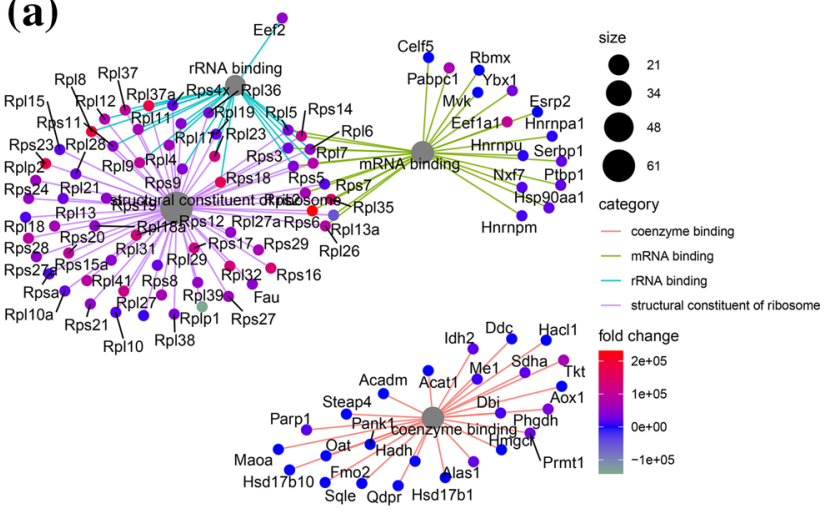

(c)

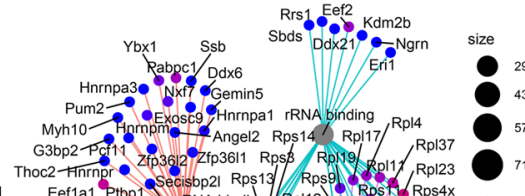

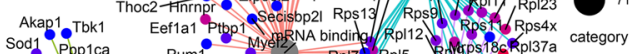

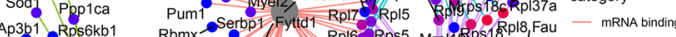

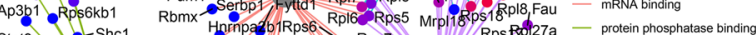

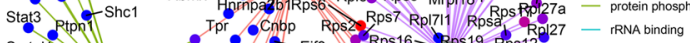

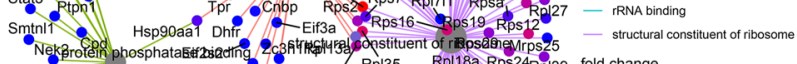

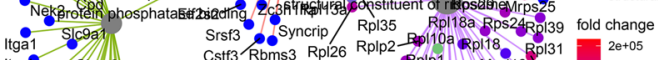

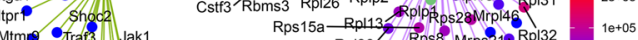

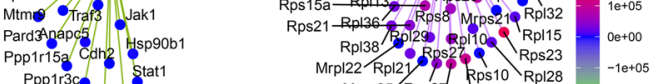

Strn3 Pik3r2

Rps20 Rpl41 (b)

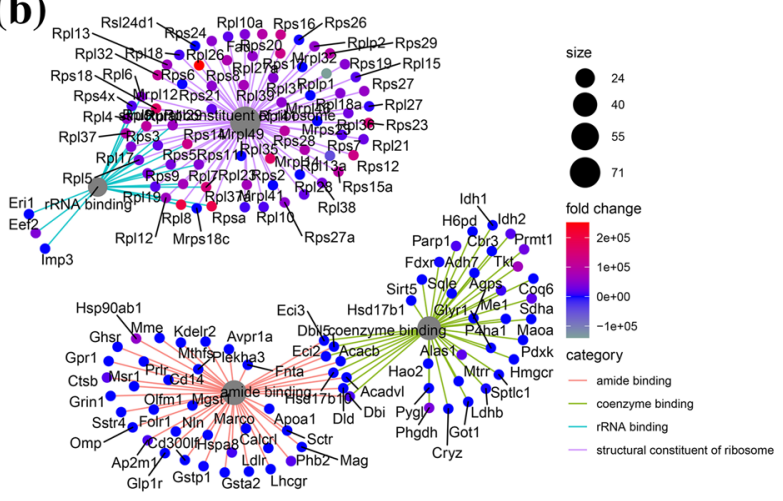

(d)

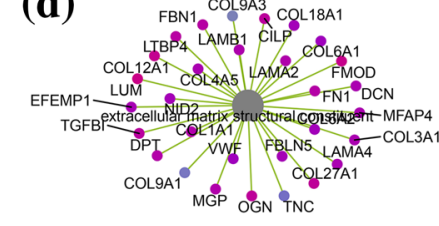

- dynein light chain binding

- extracellular matrix structural constituent

- microtubule motor activity

size

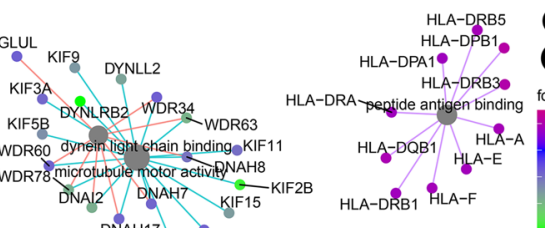
DNAH17 KIF2C KIFC3

Fig. 4 Top 4 terms from the GO MF enrichment analysis. a DEHP-exposure group. b MEHP-exposure group. c TOTM-exposure group. d NOA group. The size of the dot represents counts, and the colour of the dot represents $P$ value

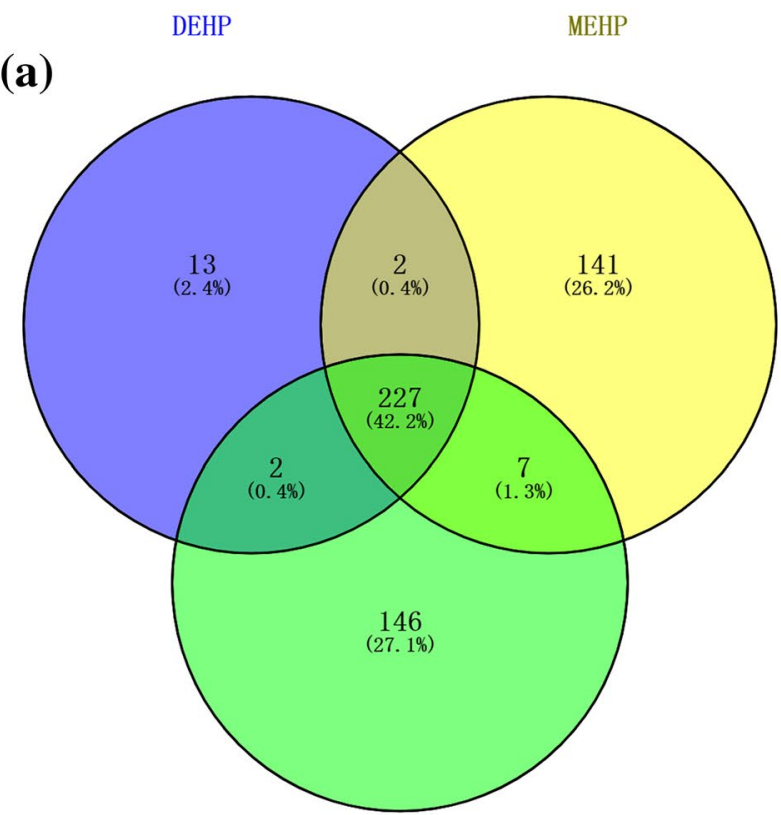

TOTM

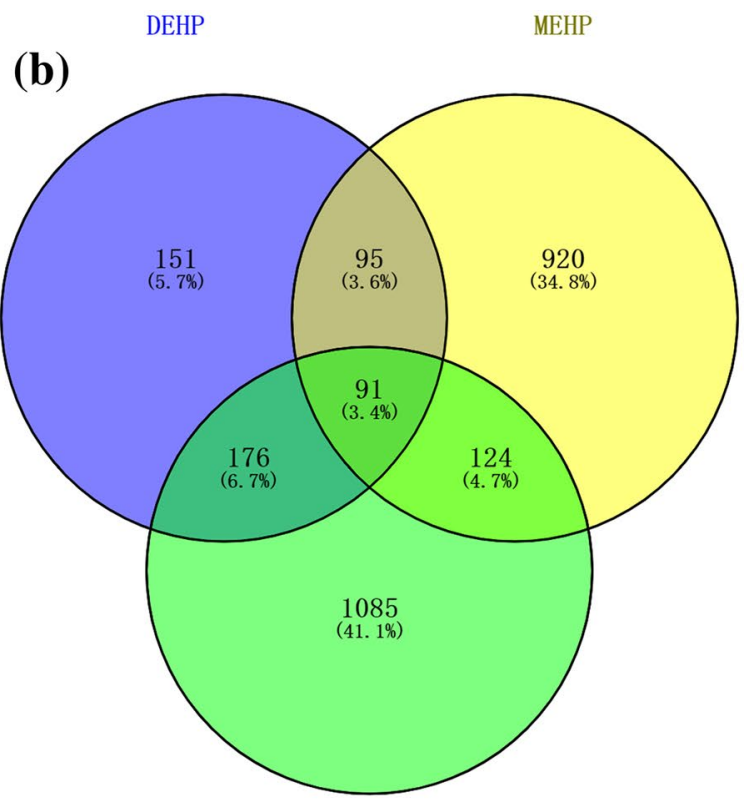

TOTM

Fig. 5 Venn diagram of overlapping differentially expression of RNA-seq data in three plasticizer-exposure groups $(\mid \operatorname{logFCl}>1$ and padj $<0.05)$ 
(a)

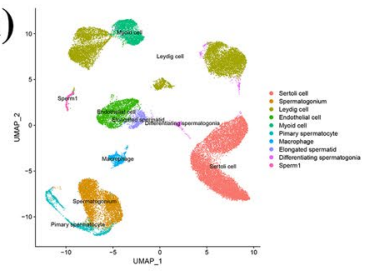

(d)

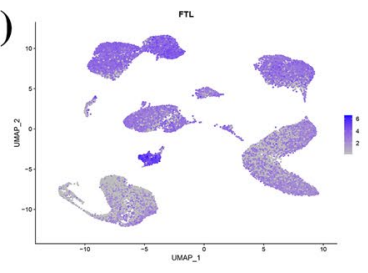

(b)

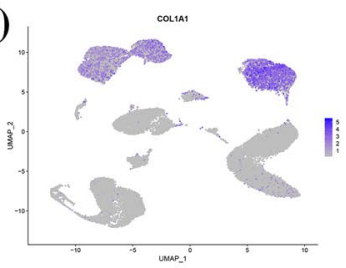

(e)

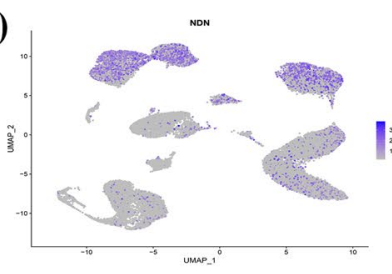

(c)

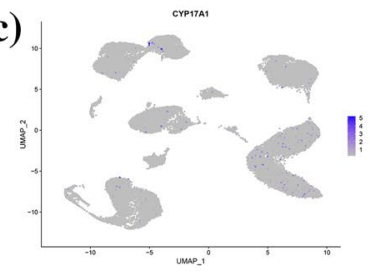

(f)

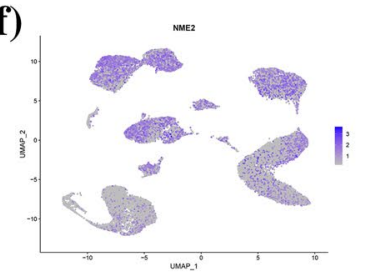

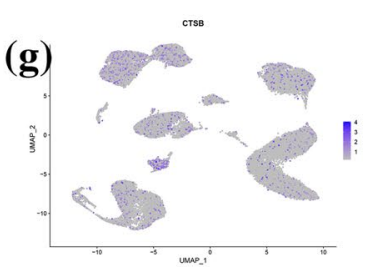

(h)

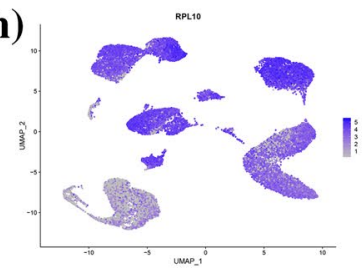

Fig. 6 Overview of the single cells from testis tissue samples. a The cell types identified by marker genes. UMAP plot of the marker genes. b-j Expression of co-expression genes for Sertoli cell, sper-

the aetiology of most NOA remains unclear. The continuous accumulation of EDCs in the human body undoubtedly causes irreversible harm to the male reproductive system (Cui et al. 2020). Plasticiser, a polymer auxiliary that is widely used in industrial production, can be found in food packaging, cosmetics and medical equipment. Previous studies confirmed that the damaging effect of plasticisers on the male reproductive system is intergenerational. Exposure to DEHP during lactation or pregnancy can damage the development of the male reproductive system of F1 offspring. A recent study evaluated sperm quality associations with urinary phthalate metabolites amongst 660 Chinese adult men. Results show that elevated urinary phthalate metabolites may affect semen quality.

The present study was based on the integrated analysis of single-cell RNA-Seq datasets and RNA-Seq datasets from GEO, which included testis samples from patients with NOA and OA and healthy adults and rats. We aimed to associate NOA with plasticiser exposure during pregnancy. After searching DEGs, we identified hundreds of genes whose expression was significantly altered in patients with NOA and rats with plasticiser exposure during pregnancy. Then, we first filtered nine co-expressed genes, including TKT, NME2, CTSB, RPL10, FTL, COL1A1, CYP17A1, NDN and KIF11. Then, we performed single-cell analysis on nine co-expressed genes and finally found three specific genes.
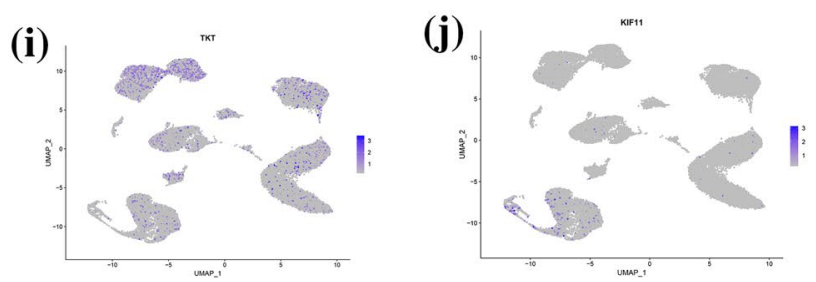

matogonium, Leydig cell, endothelial cell, myoid cell, primary spermatocyte, macrophage, elongated spermatid, differentiating spermatogonia and sperm

We found that KIF11 was significantly downregulated in patients with NOA and the plasticiser exposure group. Miki et al. found that the KIF11 protein is expressed in spermatocytes (Hara-Yokoyama et al. 2019). Besides, the immunolocalisation of KIF11 suggests the low expression in Sertoli cells. We analysed hundreds of thousands of cells in testis tissue and examined the overexpression of KIF11 in spermatogonium and primary spermatocyte. Therefore, we speculated that KIF11 played a key role in spermatogenesis. Besides, the downregulation of KIF11 could be one of the key factors that link NOA with plasticisers. A large proportion of KIF11 was correlated with the NOA led by plasticisers, which improved our understanding of NOA occurrence and helped in the development of therapeutic targets.

Testis tissues are mixtures of different compartments, e.g. Sertoli, Leydig, endothelial and myoid cells, which provide a good growth environment for sperm production and maturation. CYP17A1 is a steroidogenesis-related gene (Molaie et al. 2019). Testosterone is synthesised by Leydig cells, and the gene responsible for testosterone synthesis and cholesterol transport in testis is CYP17A1. CYP17A1 is one of the important genes in the microenvironment of sperm production (Yu et al. 2020). Sperm capacitation/acrosome reaction and testosterone biosynthesis occur in Leydig cells. We performed single-cell analysis and IHC detection and found that CYP17A1 was upregulated in patients with 
(a)
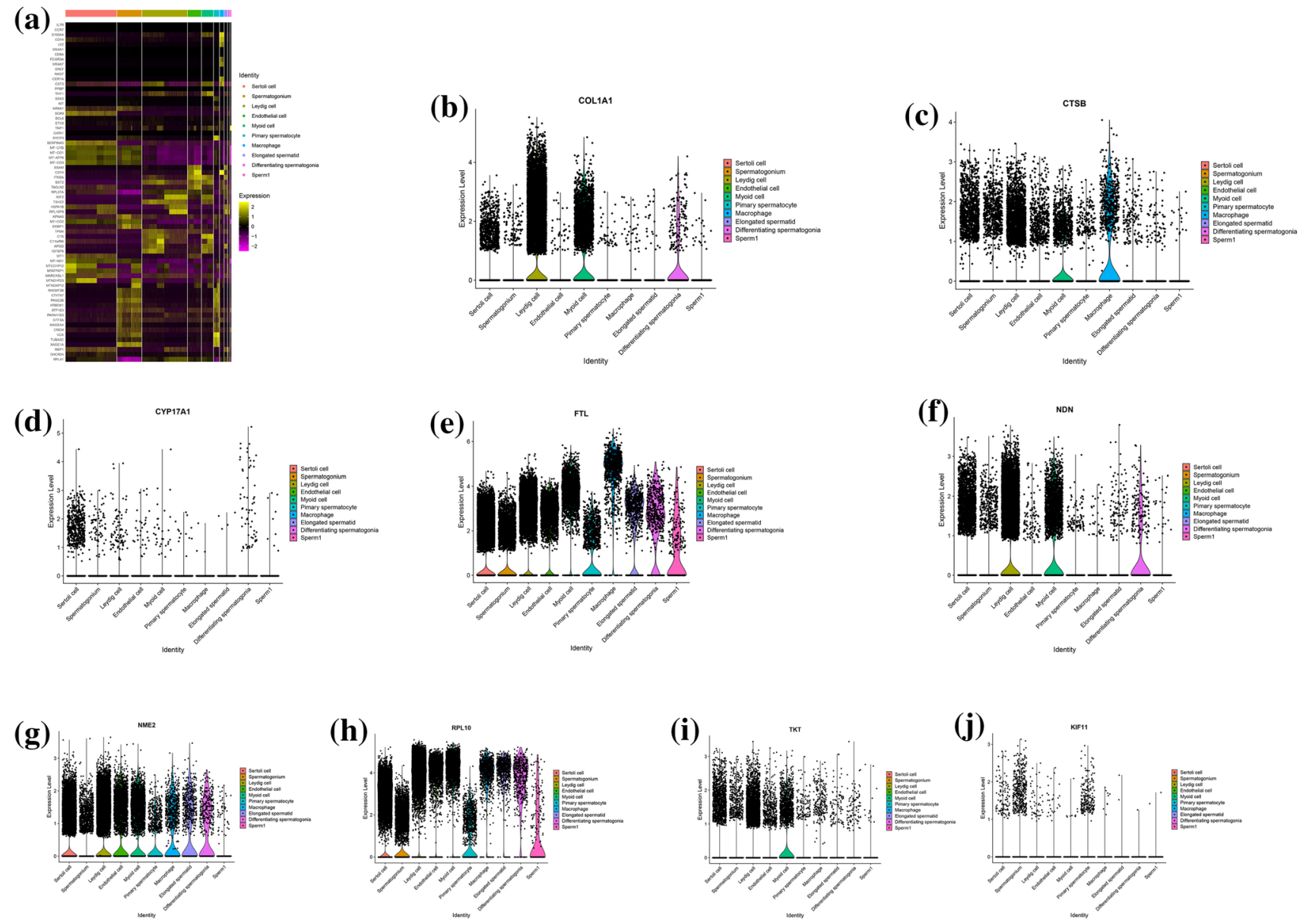

Fig. 7 a A heatmap showing the gene expression in different types of cells within testis tissues, using single-cell RNA-seq data from GSE124263. b-j violin plot chart showing the expression of nine co-

NOA and rats with plasticiser exposure. Our results showed that plasticisers might also damage sperm by affecting the function of Leydig cells to reduce testosterone production. Plasticisers may directly impair spermatogenesis and sperm maturation. Further study confirmed that promoter polymorphism may signify a genetic risk factor for male infertility (Rahali et al. 2020).

COL1A1 is a key gene implicated in epididymal immunoregulation, inflammation and fibrosis (Wijayarathna et al. 2020). The downregulation of COL1A1 in testis tissue often indicates the damage of immune system, inflammation or fibrosis (Morgan et al. 2010). By searching DEGs, the upregulation of COL1A1 was found in patients with NOA and F1 male rat offspring with plasticiser exposure. Our findings also suggested that the destruction of sperm quality in male rat offspring was associated with inflammation, fibrosis or immune damage. The elucidation of the mechanisms of immune damage, inflammation and fibrosis might improve our understanding of NOA and the reproductive damage of plasticisers.

expression genes in different types of cells, using sc-RNA-seq data from GSE124263

After the enrichment analysis of DEGs, we found that plasticiser was involved in a diversity of functions, such as sterol biosynthetic and metabolic processes, cholesterol biosynthesis, metabolic process and ribosome biogenesis, and participated in a series of physiological and pathological processes, including RNA binding, coenzyme binding and amide binding. Besides, the GO pathway analysis was conducted on the DEGs of NOA, and results showed that the functions and pathway genes activated were predominantly related to spermatid development and differentiation, fertilisation and germ cell development. Figure 11 shows the pathway related to metabolism. A direct comparison of cells with high and low expression levels of CYP17A1, COL1A1 and KIF11 was performed on the basis of the single-cell sequencing. As shown in Fig. 11, apoptotic process, cell cycle, toxic substance binding and identical protein binding were the enriched pathways. Recent studies showed that cell cycle and apoptosis regulation are associated with NOA. The percentage of apoptotic cells differentiated from patients with NOA was higher than those from normal ones. Besides, 
(a)
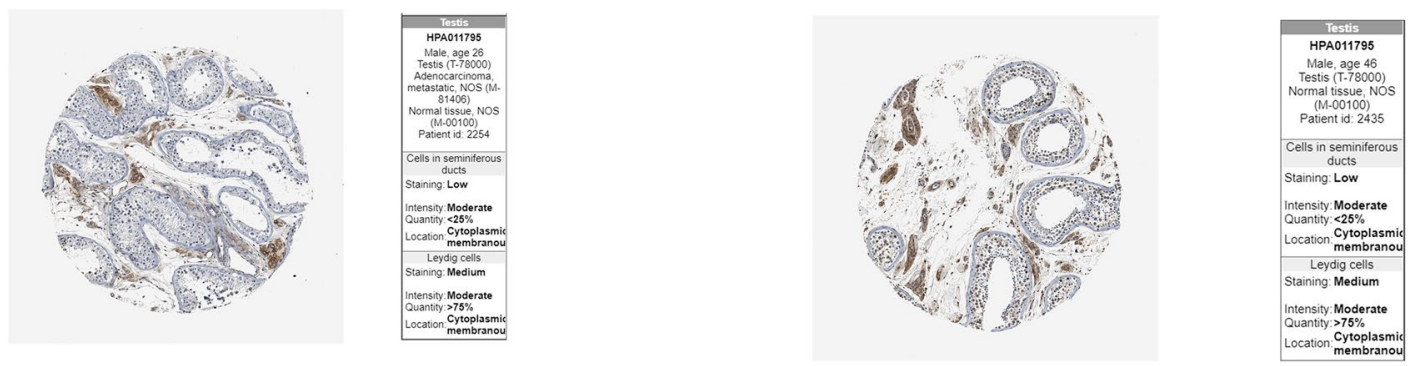

(b)
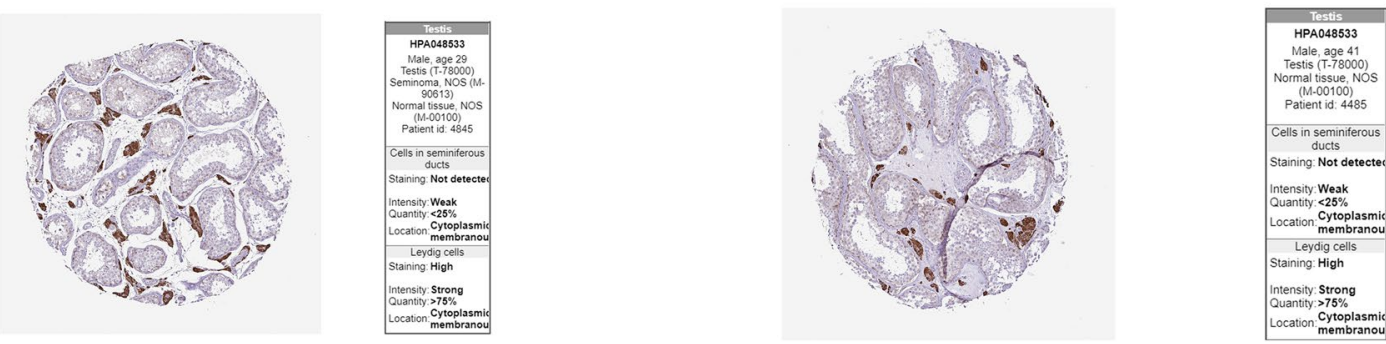

(c)
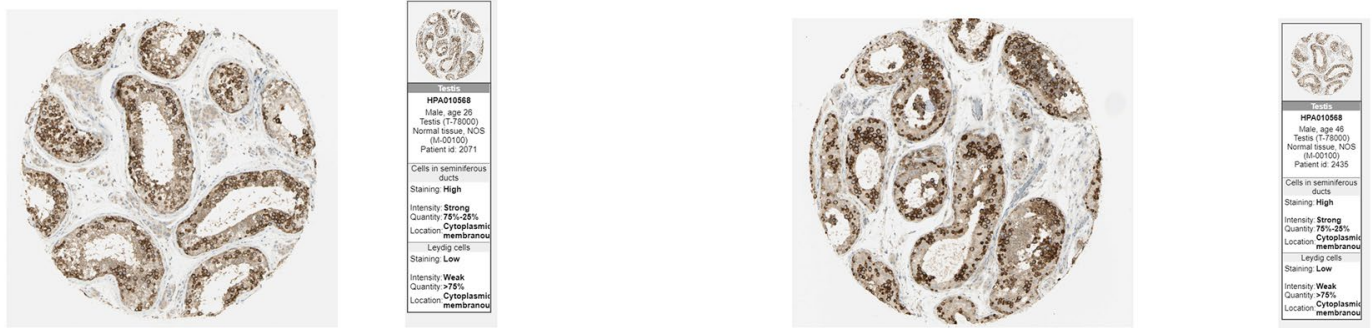

(d)
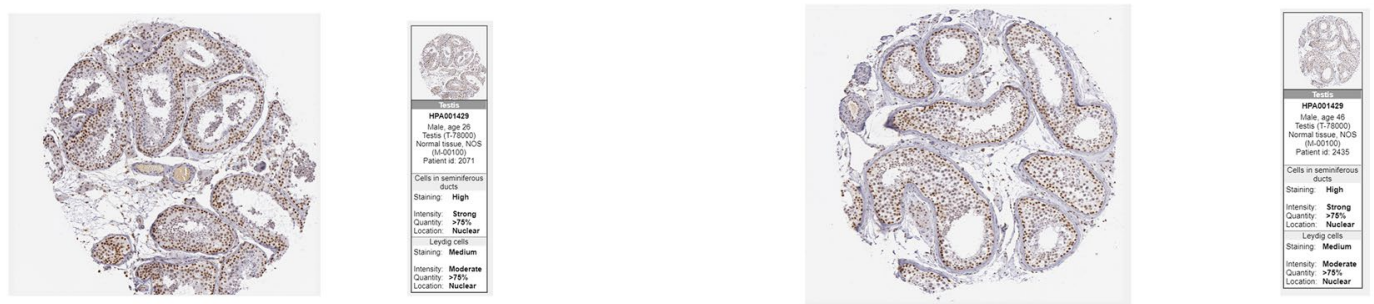

Fig. 8 Immunohistochemical images of four proteins $\mathbf{a}$ col1a1, $\mathbf{b}$ cyp17a1, $\mathbf{c}$ kif11 and $\mathbf{d} \mathrm{nbn}$

the DEGs of patients with NOA were involved in cell cycle and regulation of apoptosis. These defects might be related to the genetic causes of male infertility. A previous study confirmed that exposure to plasticisers during pregnancy leads to the activation of peroxisome proliferator-activated receptors, increased fatty acid oxidation and reduction in the ability to cope with the augmented oxidative stress, resulting in reproductive organ malformations, reproductive defects and decreased fertility. 
(a)

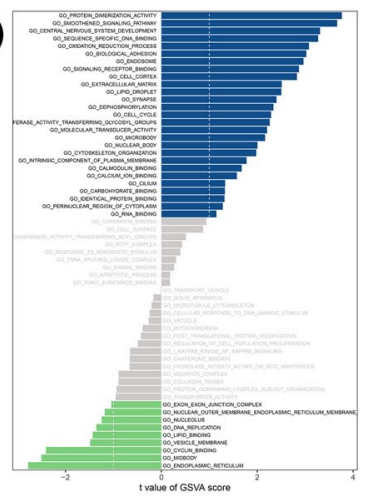

(b)

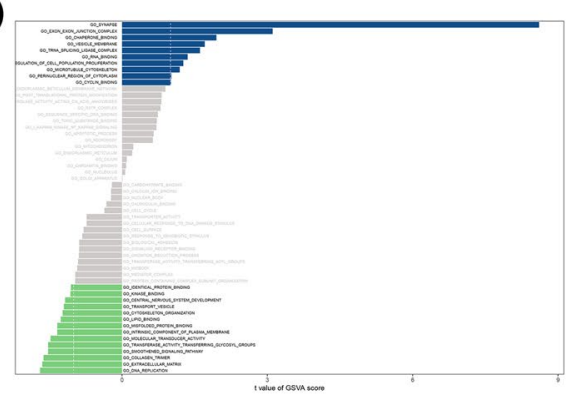

(c)

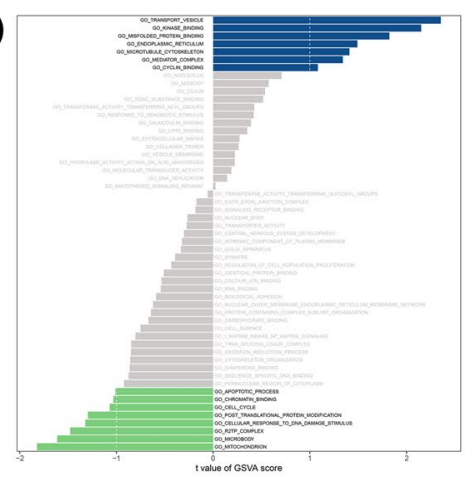

(d)

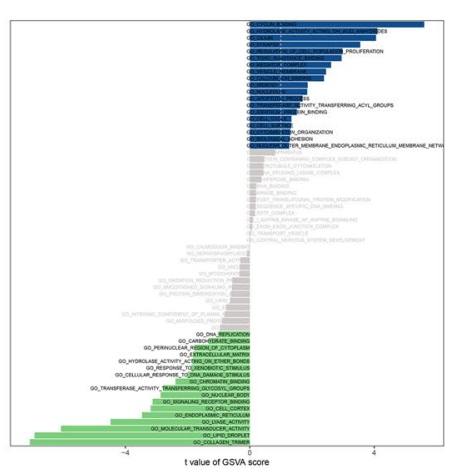

(e)

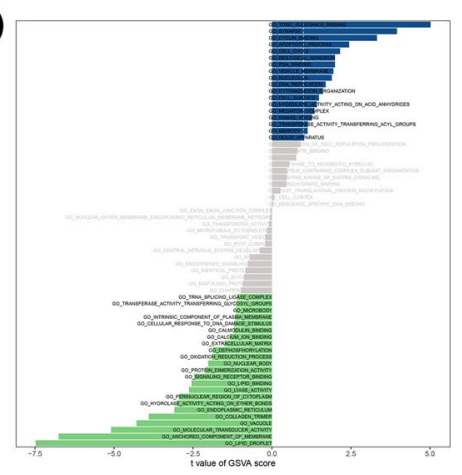

Fig. 9 GSVA analysis in human testis tissue samples. a GSVA analysis of the GO pathways between COL1A1 lowly expressing Leydig cells and COL1A1 highly expressing Leydig cells. b GSVA analysis of the GO pathways between COL1A1 lowly expressing sertoli cells and COL1A1 highly expressing sertoli cells. c GSVA analysis of the GO pathways between CYP17A1 lowly expressing sertoli cells and

In summary, exposure to plasticisers during pregnancy can affect the reproductive system of F1 male rat offspring. Besides, the long-term exposure to plasticisers during pregnancy is likely to be an important factor in male NOA. We explored DEGs and revealed the critical genes that might regulate cholesterol metabolism, transcription and protein translation in testis cells. In addition, single-cell RNA-seq
CYP17A1 highly expressing sertoli cells. d GSVA analysis of the GO pathways between KIF11 lowly expressing primary spermatocyte and KIF11 highly expressing primary spermatocyte. e GSVA analysis of the GO pathways between KIF11 lowly expressing spermatogonia and KIF11 highly expressing spermatogonia

had relatively good performance in distinguishing the molecular characteristics in each cell type. With the help of single-cell analysis, we aimed to figure out the expression of all co-downregulated and co-upregulated DEGs in different testis cells. Combined with IHC results, COL1A1, CYP17A1 and KIF11 were found to have a strong correlation in exploring the link between exposure to plasticisers 
Fig. 10 The Venn diagram demonstrated that COL1A1, CYP17A1 and KIF11 are not involved in the DEGs of GSE108194 dataset and GSE7755 dataset

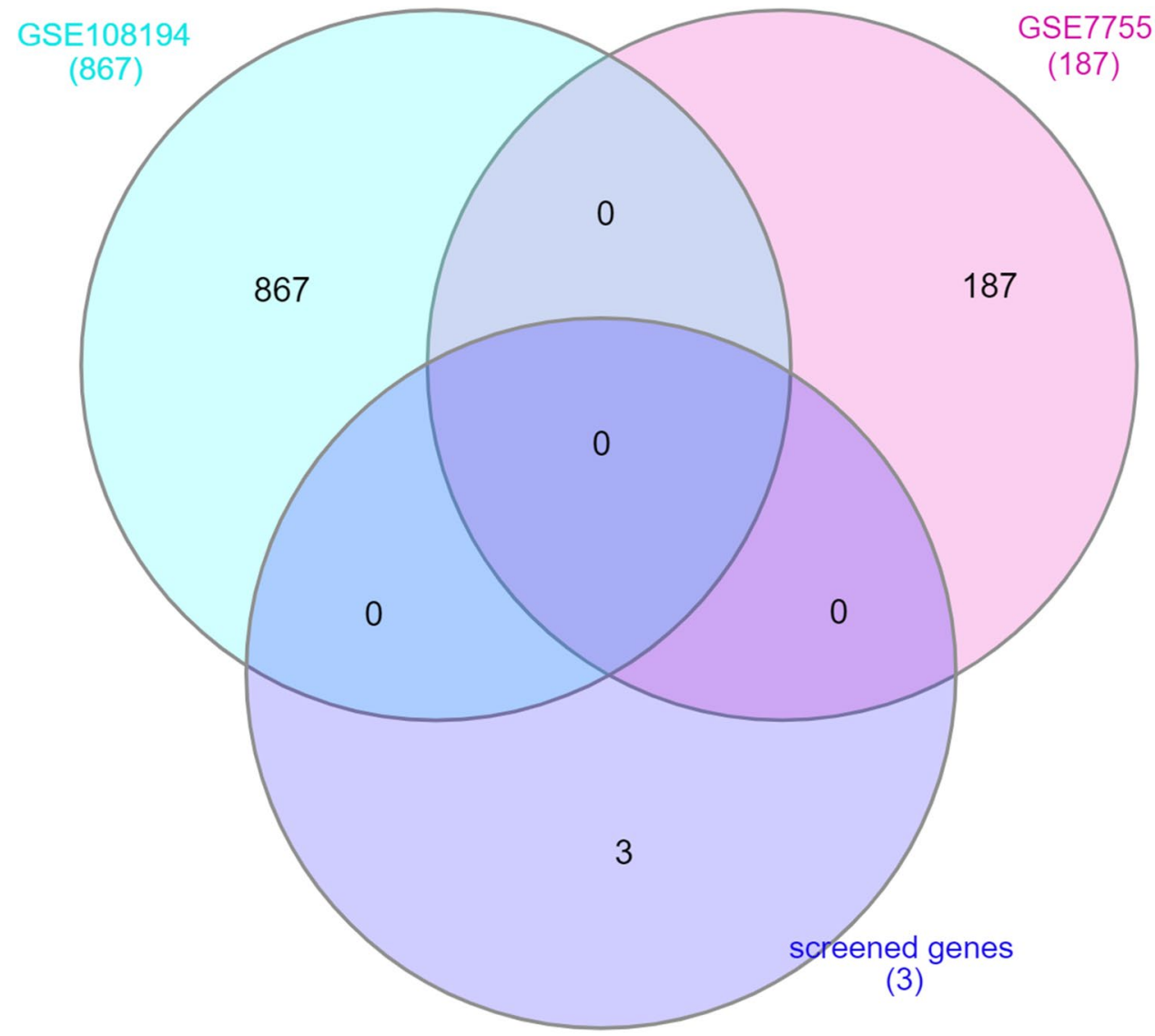

during pregnancy and human NOA. In addition, we performed the validation analysis based on the dataset of cryptorchidism and human oligospermia. The results showed that COL1A1, CYP17A1 and KIF11 are not correlated with other reproductive disorders, such as cryptorchidism and human oligospermia. Furthermore, we speculated that plasticisers might induce the impairment of the structure of seminiferous tubules and spermatogenesis, which might be attributed to the imbalance of testosterone. The damage of immune system, inflammation or fibrosis might also be involved in this impairment. Given the prevalence of plasticisers in our atmosphere and environment and the impairment of male reproductive system, we suggest their replacement. With increasing efforts to develop viable replacement compounds, rigorous leaching, toxicity and impact assessment studies are needed before alternative plasticisers can be adopted as viable replacements. 

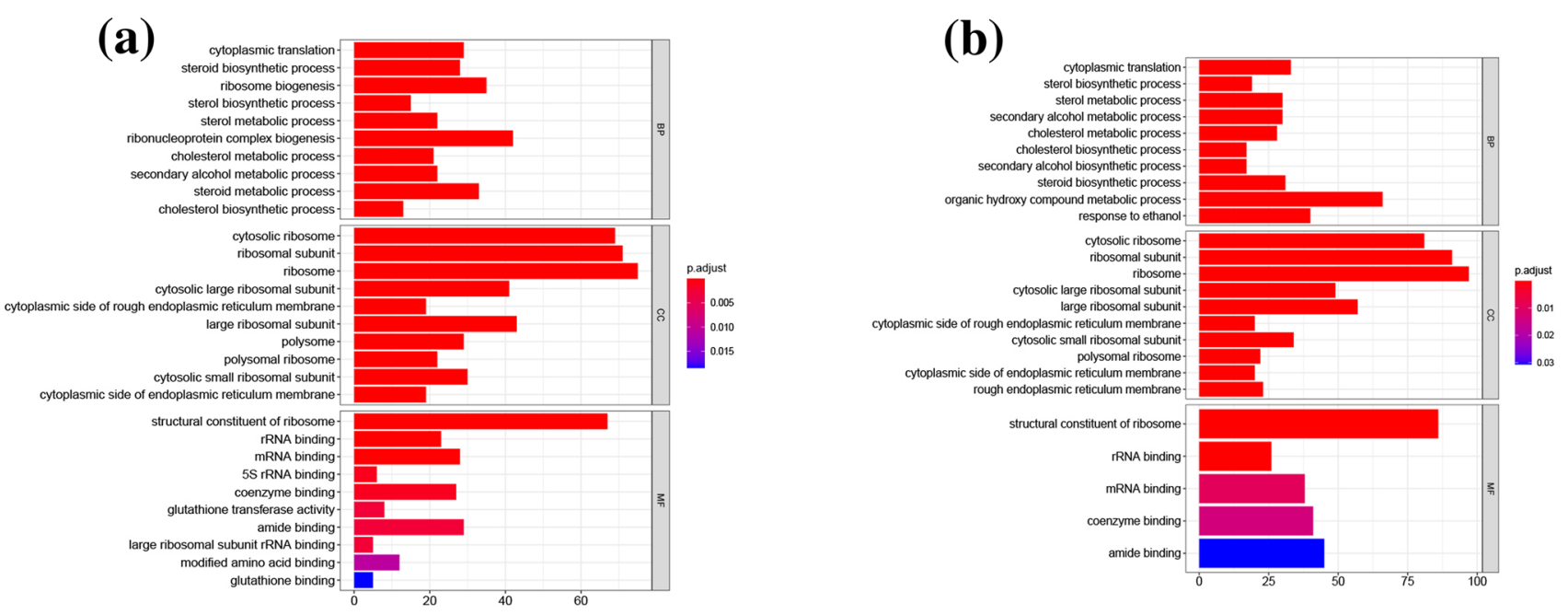

(c)

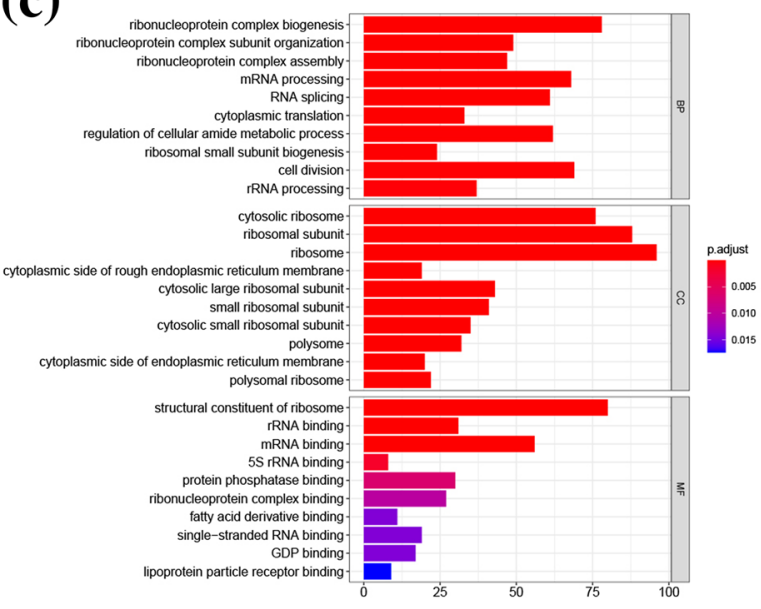

(d)

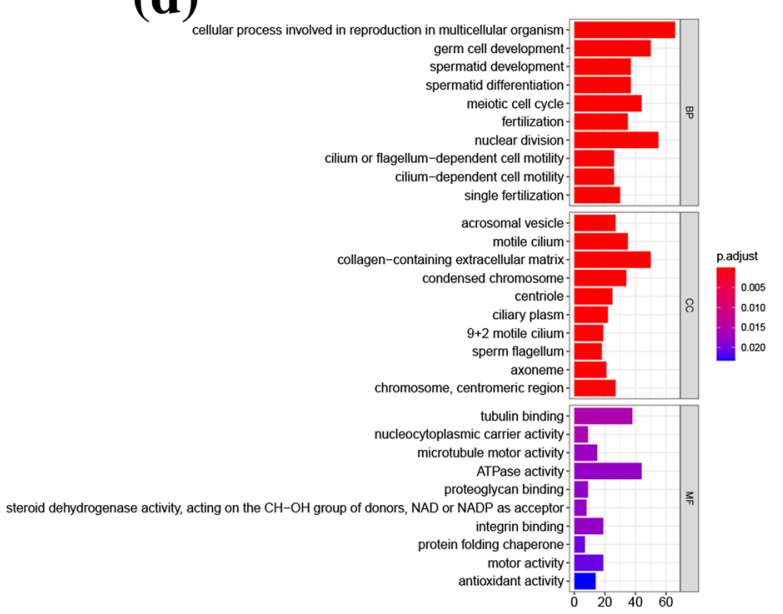

Fig. 11 Top terms from the GO BP, CC and MF enrichment analysis. A DEHP-exposure group. B MEHP-exposure group. C TOTM-exposure group. D NOA group. The size of the dot represents counts, and the colour of the dot represents $P$ value

Funding This work was supported by the National Natural Science Foundation of China (81972386, 81672531 to Chao Qin).

Data Availability The datasets generated during and/or analysed during the current study are available from the corresponding author on reasonable request.

\section{Declarations}

Conflict of interest We declare that we have no financial and personal relationships with other people or organisations that can inappropriately influence our work, there is no professional or other personal interest of any nature or kind in any product, service and/or company that could be construed as influencing the position presented in, or the research of "Comprehensive analysis of the association between human non-obstructive azoospermia and plasticizers: via single-cell RNA sequencing and traditional RNA sequencing".

Open Access This article is licensed under a Creative Commons Attribution 4.0 International License, which permits use, sharing, adaptation, distribution and reproduction in any medium or format, as long as you give appropriate credit to the original author(s) and the source, provide a link to the Creative Commons licence, and indicate if changes were made. The images or other third party material in this article are included in the article's Creative Commons licence, unless indicated otherwise in a credit line to the material. If material is not included in the article's Creative Commons licence and your intended use is not permitted by statutory regulation or exceeds the permitted use, you will need to obtain permission directly from the copyright holder. To view a copy of this licence, visit http://creativecommons.org/licenses/by/4.0/. 


\section{References}

I Al-Saleh 2019 The relationships between urinary phthalate metabolites, reproductive hormones and semen parameters in men attending in vitro fertilization clinic Sci Total Environ 658982995 https://doi.org/10.1016/j.scitotenv.2018.12.261

R Barakat T Seymore PP Lin CJ Park CJ Ko 2019 Prenatal exposure to an environmentally relevant phthalate mixture disrupts testicular steroidogenesis in adult male mice Environ Res 172194201 https://doi.org/10.1016/j.envres.2019.02.017

Y Cui 2020 Dibutyl phthalate-induced oxidative stress, inflammation and apoptosis in grass carp hepatocytes and the therapeutic use of taxifolin Sci Total Environ https://doi.org/10.1016/j.scitotenv. 2020.142880

MM Dobrzynska EJ Tyrkiel 2019 The effect of preconceptional exposure of F0 male mice to di(2-ethylhexyl)phthalate on the induction of reproductive toxicity in F2 generation Drug Chem Toxicol 42 $546551 \mathrm{https}: / /$ doi.org/10.1080/01480545.2018.1468448

M Golshan SMH Alavi 2019 Androgen signaling in male fishes: examples of anti-androgenic chemicals that cause reproductive disorders Theriogenology $1395871 \mathrm{https} / / /$ doi.org/10.1016/j.theri ogenology.2019.07.020

M Hara-Yokoyama 2019 KIF11 as a potential marker of spermatogenesis within mouse seminiferous tubule cross-sections J Histochem Cytochem 67813824 https://doi.org/10.1369/0022155419871027

R Hauser 2008 Urinary phthalate metabolites and semen quality: a review of a potential biomarker of susceptibility Int J Androl 31 112117 https://doi.org/10.1111/j.1365-2605.2007.00844.x

TK Kasonga MAA Coetzee I Kamika VM Ngole-Jeme MN Benteke Momba 2021 Endocrine-disruptive chemicals as contaminants of emerging concern in wastewater and surface water: a review J Environ Manag 277111485 https://doi.org/10.1016/j.jenvman. 2020.111485

S Kuroda 2020 Genetic disorders and male infertility Reprod Med Biol $19314322 \mathrm{https} / / /$ doi.org/10.1002/rmb2.12336

J Li 2021 Effects of butyl benzyl phthalate exposure on Daphnia magna growth, reproduction, embryonic development and transcriptomic responses J Hazard Mater 404124030 https://doi. org/10.1016/j.jhazmat.2020.124030

BE Lucas C Fields N Joshi MC Hofmann 2012 Mono-(2-ethylhexyl)phthalate (MEHP) affects ERK-dependent GDNF signalling in mouse stem-progenitor spermatogonia Toxicology 2991019 https://doi.org/10.1016/j.tox.2012.04.011

L Minguez-Alarcon 2018 Secular trends in semen parameters among men attending a fertility center between 2000 and 2017: identifying potential predictors Environ Int $12112971303 \mathrm{https} / / /$ doi.org/10.1016/j.envint.2018.10.052
S Molaie 2019 Dietary trans and saturated fatty acids effects on semen quality, hormonal levels and expression of genes related to steroid metabolism in mouse adipose tissue Andrologia 51 e13259 https://doi.org/10.1111/and.13259

CC Morgan NB Loughran TA Walsh AJ Harrison MJ O'Connell 2010 Positive selection neighboring functionally essential sites and disease-implicated regions of mammalian reproductive proteins BMC Evol Biol 1039 https://doi.org/10.1186/1471-2148-10-39

H Okada A Tajima K Shichiri A Tanaka K Tanaka I Inoue 2008 Genome-wide expression of azoospermia testes demonstrates a specific profile and implicates ART3 in genetic susceptibility PLoS Genet 42 e26 https://doi.org/10.1371/journal.pgen.00400 26.PMID: $18266473 ;$ PMCID:PMC2233677

D Rahali 2020 Spermatogenesis and steroidogenesis disruption in a model of metabolic syndrome rats Arch Physiol Biochem https://doi.org/10.1080/13813455.2020.1812665

R Rahban S Nef 2020 Regional difference in semen quality of young men: a review on the implication of environmental and lifestyle factors during fetal life and adulthood Basic Clin Androl 3016 https://doi.org/10.1186/s12610-020-00114-4

IA Sheikh 2016 Human sex hormone-binding globulin as a potential target of alternate plasticizers: an in silico study BMC Struct Biol 1615 https://doi.org/10.1186/s12900-016-0067-3

MK Stewart DM Mattiske AJ Pask 2020 Exogenous oestrogen impacts cell fate decision in the developing gonads: a potential cause of declining human reproductive health Int J Mol Sci https://doi.org/ 10.3390/ijms21218377

X Sun 2020 Effects of the environmental endocrine disruptors di2-ethylhexyl phthalate and mono-2-ethylhexyl phthalate on human sperm function in vitro Reprod Fertil Dev 32629636 https://doi. org/10.1071/RD19164

YX Wang 2019 Seminal plasma metabolome in relation to semen quality and urinary phthalate metabolites among Chinese adult men Environ Int 129354363 https://doi.org/10.1016/j.envint. 2019.05.043

R Wijayarathna 2020 Region-specific immune responses to autoimmune epididymitis in the murine reproductive tract Cell Tissue Res 381351360 https://doi.org/10.1007/s00441-020-03215-8

W Yu 2020 Protective role of wogonin against cadmium induced testicular toxicity: involvement of antioxidant, anti-inflammatory and anti-apoptotic pathways Life Sci https://doi.org/10.1016/j. lfs. 2020.118192

Publisher's Note Springer Nature remains neutral with regard to jurisdictional claims in published maps and institutional affiliations. 